\title{
Estimation and Solution of Models with Expectations and Structural Changes*
}

\author{
Mariano Kulish ${ }^{\dagger}$ and Adrian Pagan ${ }^{\ddagger}$
}

February 29, 2016

\begin{abstract}
In this paper we develop solutions for linearised models with forward looking expectations and structural changes under a variety of assumptions regarding agents' beliefs about those structural changes. For each solution we show how its associated likelihood function can be constructed by using a "backward-forward" algorithm. We illustrate the techniques with two examples. The first considers an inflationary program in which beliefs about the inflation target evolve differently from the inflation target itself and the second applies the techniques to estimate a New-Keynesian model through the Volcker disinflation. We compare our methodology with the alternative in which structural change is captured by switching between regimes via a Markovswitching process. We show that our method can produce accurate results much faster than the MS method as well as being easily adapted to handle beliefs departing from reality.
\end{abstract}

JEL: C13, C32, C63, E52

Keywords: Expectations, structural change, DSGE estimation, maximum likelihood, disinflation, announcements.

${ }^{*}$ We thank Adam Cagliarini, Efrem Castelnuovo, Alex Heath, Greg Kaplan, Gordon Menzies, James Morley, Michael Plumb and Frank Schorfheide for useful discussions. We thank seminar participants at ANU, Universidad Torcuato Di Tella, Reserve Bank of Australia and at the Dynare Conference at SUFE for feedback. We also wish to thank the editor and anonymous referees for useful comments. Research for the first author was supported under Australian Research Council's Linkage Projects funding scheme LP140101020. Work on this paper by the second author was supported by Australian Research Council Dicovery Grant DP160102654.

${ }^{\dagger}$ School of Economics, UNSW and CAER, m.kulish@unsw.edu.au

${ }^{\ddagger}$ School of Economics, Faculty of Arts and Social Sciences, University of Sydney and Melbourne Institute of Applied Economic and Social Research, University of Melbourne, apagan@unimelb.edu.au 


\section{Introduction}

Standard solution methods for linear rational expectations models, such as Anderson (1997), Blanchard and Kahn (1980), Binder and Pesaran (1995), King and Watson (1998), Klein (2000), Sims (2001) and Uhlig (1999), deal with the case where the parameters of the structural model are constant. These methods are at the heart of likelihood-based estimation of such models. In practice, the magnitude of changes in the properties of observable variables is often used to help define sub-samples for which a time-invariant structure seems valid, and estimation is then done with these sub-samples. For instance, based on the estimates of steady-state inflation in each of two sub-samples, the analysis of Lubik and Schorfheide (2004) is based on the assumption that the target inflation rate in the United States - like other structural parameters - stayed constant in the preVolcker years, but then possibly shifted in the early 1980s. This example points to the fact that the structural change may come from policy makers setting new targets that become embedded into the economic system as parameters. Other examples would be the announcements of new tax rates and changes in an inflation target. There might be many of the latter. Figure 1 shows this for the inflation targets that the Central Bank of Chile announced and later implemented over the course of nearly two decades when aiming to bring down inflation. Thus it is important when developing methods that allow for the interaction of expectations and structural change to recognize that there may be many different structural models, each relevant to a particular period of time. In what follows we refer to these as structures.

Findings of structural instabilities seem to apply to many models of macroeconomic aggregates. While we cannot do justice to the complete literature, one can point to the work of Clarida et al. (2000) who find a significant difference in the way monetary policy was conducted pre- and post-late 1979 in the United States; Ireland (2001) who detects shifts in the discount factor; Inoue and Rossi (2011) who show that the Great Moderation was due to changes in shock volatilities as well as policy and private sector parameters; Primiceri (2005) who find that both systematic and non-systematic monetary policy have changed during the last 40 years; and Stock and Watson (2007) who provide evidence of changes in the variance of shocks to trend inflation. 
We make three important extensions to the existing literature which deals with the type of issues discussed in this paper. First we allow for structural changes that are either unanticipated or anticipated. Second, agents' beliefs about when the structural changes can occur may differ from the actual dates, at least for a period of time. In a recent paper, Cagliarini and Kulish (2013) dealt with the case of anticipated structural change where beliefs coincide with reality, so our work is an obvious extension of that paper. Third, and more importantly, our solution takes the form of a time-varying coefficient VAR so it can be used to formulate a likelihood that can be employed to estimate the parameters of the structural models, along with the dates at which any structural breaks occur. This contrasts with Cagliarini and Kulish (2013) who formulate the problem as a system $A y=b$, where the vector $y$ contains the entire expected path from the time agents know of the future change to the time the change takes place. Because one can check the rank of $A$ for any given application, that solution is particularly useful for assessing non-existence, uniqueness and multiplicity of the solution paths. But because the solution involves a stacking method, it is not in a form that can be taken easily to the data; that is, it is not easy to exploit to find the likelihood.

As noted above, the new formulation of this paper allows for a difference between beliefs and reality to exist for a period of time either before or after any structural changes occur. This extension allows us to capture situations in which structural changes take place or policy announcements are carried out but these are not necessarily fully incorporated into expectations formation. An example of this might be the US productivity slowdown of the early 1970s, where the consequences of such a structural change went unnoticed temporarily. We place few restrictions on the set of possible beliefs. In every case, however, the reduced-form solution has an associated likelihood function. So, while the cases that can be solved for are quite general, the utility of this generalisation lies not just in solving for arbitrary beliefs, but also in the possibility of taking a given set of beliefs to the data. Then, such data may narrow down the set of beliefs, rejecting one in favour of another. This testing aspect is possible precisely because of the explicit derivation of a likelihood.

In the basic case it is assumed that expectations are formed in such a way as to be consistent with whatever structure (model) holds at each point in the sample. This is analysed in Section 3 by looking at cases where the structural changes are either unknown 
in advance or where there is some foresight about them. In the scenario where there is just a single break the second structure (model) holds at some given future date and, because agents know what that date is, they factor it into the formation of their expectations before the actual time at which the change occurs. Exploiting this fact leads to the time-varying VAR solution mentioned above. Section 3 also deals with the situation where beliefs about the structural change can be different from the truth (reality). Thus, if one thinks of a single structural change in the sample, expectations may be based on the first period model for some time into the second period. Of course, eventually it seems reasonable (though not necessary) to think that beliefs must centre upon the second period model.

In the context of estimating and solving dynamic stochastic models with expectations, some knowledge of the structural changes that might have taken place in-sample allows us to increase the number of observations that are usable in estimation, and therefore has the potential for improving the quality of the estimation. Therefore this paper makes a significant methodological contribution by providing easy-to-implement tools to accomplish maximum likelihood estimation of dynamic stochastic economies with structural changes under a variety of assumptions regarding expectations formation.

The paper is organized as follows. The next section reviews the Binder and Pesaran (1997) solution procedure for models with forward-looking expectations, which is essentially an implementation of the method of undetermined coefficients. As mentioned previously, Section 3 then extends the solution to situations of structural change and derives the likelihood for the implied model. Section 4 introduces two examples. In the first example we focus on impulse responses in the context of a scenario where policy increases an inflation target. Specifically, we compare the responses when this policy is credibly anticipated with the case when it is not. In the second example we estimate characteristics of the Volcker disinflation using expectations data, as well as aggregate data for the United States for the period 1957 to 2008.

Structural changes raise two questions - where do they occur and what is their magnitude? These questions arise both for agents and for the econometrician. In our analysis agents know when a change takes place (or at least within a certain period of time afterwards) and also the magnitudes of the changes, but the econometrician has to discover these features. Section 3 looks at our Backward-Forward (BF) method for discovering this 
information. Section 4 gives examples of its use while section 5 compares the performance of the BF method to an alternative one involving Markov-switching (MS) models, where agents know the probability that a structural change will occur as well as its magnitude. The difference between the two approaches is that in the BF method the focus is on the points in the sample where parameter breaks occur rather than a probability. In general, a break will mean that a new structural model (or regime) operates. But this is not always so. Instead, a break could feature a return to a regime rather than a new one i.e. the regimes recur. This is what is done in the MS approach. Regimes recur with a certain probability. When there is a return to a regime this can still be classified as a structural break and may mean that the number of breaks can exceed the number of regimes.

We use two experiments to compare the approaches. In the first experiment there are two regimes and a single switch between them i.e. a single structural change. In the other one there are three changes. The simulated data enables us to know exactly where the structural breaks occur. Our method for identifying the time of the break does well in comparison to the MS approach for both experiments and is far faster to compute. Our discussion of these examples also points to the fact that there are advantages and disadvantages of each approach, largely connected with whether the dates of the breaks are known and how many breaks might be expected within a sample of data. Section 6 then concludes with some suggestions about extensions of the BF method to encompass learning and non-linear structures. On-line appendices provide details of the construction of the likelihood with the Kalman filter that is used in estimation, how posterior densities for the model coefficients and the date of breaks are found, and provide a fuller comparison with the MS approaches.

\section{Solution of Models with Forward-Looking Expectations and No Structural Changes}

Our solution method is a variant of Binder and Pesaran (1997). Following that paper, a linear rational expectations model of $n$ equations can be written as

$$
A_{0} y_{t}=C_{0}+A_{1} y_{t-1}+B_{0} \mathbb{E}_{t} y_{t+1}+D_{0} \varepsilon_{t},
$$


where $y_{t}$ is a $n \times 1$ vector of state and jump variables and $\varepsilon_{t}$ is a $l \times 1$ vector of exogenous variables ("shocks"). With no loss of generality we take the latter to be white noise and to have $I_{l}$ as their covariance matrix. All matrices in Equation (1) conform to the specified dimensions. ${ }^{1}$ The formulation can be generalised as in Binder and Pesaran (1997) to allow additional lags of $y_{t}$ as well as conditional expectations at different horizons and from earlier dates.

If it exists and is unique, the solution to Equation (1) will be a VAR of the form

$$
y_{t}=C+Q y_{t-1}+G \varepsilon_{t}
$$

Given that this is the solution and $\mathbb{E}_{t} \varepsilon_{t+1}=0$ we must have $\mathbb{E}_{t} y_{t+1}=C+Q y_{t}$. Substituting this into Equation (1) and re-arranging terms produces

$$
y_{t}=\left(A_{0}-B_{0} Q\right)^{-1}\left(C_{0}+B_{0} C+A_{1} y_{t-1}+D_{0} \varepsilon_{t}\right) .
$$

Now $\left(A_{0}-B_{0} Q\right)^{-1}=\left(I-A_{0}^{-1} B_{0} Q\right)^{-1} A_{0}^{-1}$ and, after defining $\Gamma \equiv A_{0}^{-1} C_{0}, A \equiv A_{0}^{-1} A_{1}$, $B \equiv A_{0}^{-1} B_{0}$ and $D \equiv A_{0}^{-1} D_{0}$, Equation (3) can be written as

$$
y_{t}=(I-B Q)^{-1}\left(\Gamma+B C+A y_{t-1}+D \varepsilon_{t}\right)
$$

Using the same logic as in the method of undetermined coefficients this must equal Equation (2), establishing the equivalences

$$
\begin{aligned}
(I-B Q)^{-1}(\Gamma+B C) & =C, \\
(I-B Q)^{-1} A & =Q, \\
(I-B Q)^{-1} D & =G .
\end{aligned}
$$

\footnotetext{
${ }^{1}$ We may need to make a distinction between the original shocks of a dynamic stochastic model, $e_{t}$, and the shocks $\varepsilon_{t}$ in Equation (1). Often $e_{t}$ are taken to be serially correlated. This can be captured by writing such a system in the form of Equation (1) with lagged values of the endogenous variables included in $y_{t}$. This means that $\varepsilon_{t}$ are the innovations to the shock processes $e_{t}$. There may be a numerical advantage to working with $e_{t}$ rather than $\varepsilon_{t}$, as that reduces the dimension of $y_{t}$ and, consequently, all the matrices involved in finding a solution. But there are conceptual advantages in using the system we work with. Our MATLAB function that computes the Binder Pesaran solution, 'smatsbp.m', does allow us to work with an $e_{t}$ that is described by a VAR process.
} 
Equation (5) implies that

$$
A-Q+B Q^{2}=0
$$

and so determines $Q$. Equation (4) implies that

$$
C=\left[\left(I-(I-B Q)^{-1} B\right]^{-1}(I-B Q)^{-1} \Gamma=(I-F)^{-1} \Lambda,\right.
$$

where $\Lambda=(I-B Q)^{-1} \Gamma, F=(I-B Q)^{-1} B$. Thus, once $Q$ is found, it is possible to derive $C$ and $G$, providing the solution to the model.

\section{Solutions with Structural Changes}

Now we wish to use the method of undetermined coefficients from the previous section to deal with structural change. To motivate what we deal with consider the following equation from Ireland (2004). In this $\pi_{t}$ is the inflation rate, $r_{t}$ is an interest rate and $x_{t}$ is an output gap. The output gap is taken to have a zero steady state value and $\bar{r}$ and $\bar{\pi}$ are the steady state values of $r_{t}$ and $\pi_{t}$.

$$
x_{t}=(\bar{r}-\bar{\pi})-\left(r_{t}-\mathbb{E}_{t} \pi_{t+1}\right)+\mathbb{E}_{t} x_{t+1}+(1-\omega)\left(1-\rho_{a}\right) a_{t}
$$

We now want to allow for the fact that $\bar{r}$ and $\bar{\pi}$ might change over time. Suppose that the structure for $t=1, . ., T_{m}-1$ has steady states of $\bar{r}^{(1)}$ and $\bar{\pi}^{(1)}$ but a new structure operates from $T_{m}$ with steady states of $\bar{r}^{(2)}$ and $\bar{\pi}^{(2)}$. Rather than distinguishing these by the superscripts we will define $\bar{r}_{t}$ and $\bar{\pi}_{t}$ as being $\bar{r}_{t}=\bar{r}^{(1)}, \bar{\pi}_{t}=\bar{\pi}^{(1)}$ for $t=1, \ldots, T_{m}-1$, while $\bar{r}_{t}=\bar{r}^{(2)}, \bar{\pi}_{t}=\bar{\pi}^{(2)}$ for $t=T_{m}, \ldots$ Of course it may be that other parameters such as $\rho_{a}$ have different values in each structure and, if so, we would need to allow them to vary as well. But these may not affect the steady states and it is the shifts in the steady states that create most of the difficulties. Later we will write the equation above as having a term $C_{0, t}=\bar{r}_{t}-\bar{\pi}_{t}$.

Now let us generalize the discussion above. First, there is a sample of data running as $t=1,2, \ldots, T$. Second, we allow for a number of structural changes over the sample period. Hence we start by assuming that the first structural change is at $T_{m}$ and the last 
is at $T_{m}^{*}$. In between $T_{m}$ and $T_{m}^{*}$ there may be a number of different structures i.e. the initial structure is replaced by a new structure at $T_{m}$, following which there may be a sequence of structures until $T_{m}^{*}$, when a final structure is in place. After $T_{m}^{*}$ no further structural changes are assumed to take place (and we will say that the structures have converged). Notice that, given these definitions, if there is just a single structural change then it begins at $T_{m}=T_{m}^{*}$, since the model after the initial one is the final model.

Figure 2 illustrates one possibility. The arrows describe the evolution of the structure. The sequence of structural changes begins in $T_{m}$ and ends in $T_{m}^{*}$. In Figure 2, just as in our later examples, $T_{m}$ and $T_{m}^{*}$ are within the sample, although nothing about our solution requires this to be the case. Further, in practice, one might also have many sequences of structural changes in the model parameters (and these could possibly overlap); it suffices to establish the solutions with a single sequence of structural changes.

A formal account of the description above follows. Formally it is being assumed that before $T_{m}$ the structure is stable at Equation (1). So, in the notation of that equation $C_{0}$ reflects the steady state in the period up to $T_{m}$. Then the new structures during $t=T_{m}, \ldots, T_{m}^{*}-1$ will be captured by

$$
A_{0, t} y_{t}=C_{0, t}+A_{1, t} y_{t-1}+B_{0, t} \mathbb{E}_{t} y_{t+1}+D_{0, t} \varepsilon_{t} .
$$

Because there are potentially a number of different steady states during this period we designate these by $C_{0, t}$. Other parameters $A_{0, t}$ etc.may also vary. Eventually from $T_{m}^{*}$ onwards there is a single structure

$$
A_{0}^{*} y_{t}=C_{0}^{*}+A_{1}^{*} y_{t-1}+B_{0}^{*} \mathbb{E}_{t} y_{t+1}+D_{0}^{*} \varepsilon_{t} .
$$

Thereafter, there are no further structural changes and Equation (8) holds into the infinite future.

To be concrete suppose there are just two structural changes in the sample. In the first interval $\left(1\right.$ to $\left.T_{m}-1\right)$ there is a model whose coefficients are $\theta=\left\{A_{0}, C_{0}, A_{1}, B_{0}, D_{0}\right\} .{ }^{2} \mathrm{In}$ the second interval $\left(T_{m}\right.$ to $\left.T_{m}^{*}-1\right)$ these change to $\bar{\theta}=\left\{\bar{A}_{0}, \bar{C}_{0}, \bar{A}_{1}, \bar{B}_{0}, \bar{D}_{0}\right\}$ i.e. $C_{0, t}=\bar{C}_{0}$

\footnotetext{
${ }^{2}$ Note that because there is only one regime between $T_{m}$ and $T_{m}^{*}-1$ we can describe $A_{0, t}, C_{0, t}, \ldots$ etc as $\bar{A}_{0}, \bar{C}_{0} \ldots$
} 
etc. and, in the final interval $\left(T_{m}^{*}\right.$ to $\left.T\right)$, to $\theta^{*}=\left\{A_{0}^{*}, C_{0}^{*}, A_{1}^{*}, B_{0}^{*}, D_{0}^{*}\right\}$. The notation in Equation (7) allows the parameters $A_{0, t}$ etc. to vary according to the time period but, in the two structural change case $A_{0, t}=\bar{A}_{0}$ etc. from $T_{m}$ to $T_{m}^{*}-1$, and, after that, the structure converges to $A_{0}^{*}$, etc. In general, when a sequence of structural changes takes place in-sample, the structural matrices are given by

$$
\left\{\left\{A_{0}, C_{0}, A_{1}, B_{0}, D_{0}\right\}_{t=1}^{T_{m}-1},\left\{A_{0, t}, C_{0, t}, A_{1, t}, B_{0, t}, D_{0, t}\right\}_{t=T_{m}}^{T_{m}^{*}-1},\left\{A_{0}^{*}, C_{0}^{*}, A_{1}^{*}, B_{0}^{*}, D_{0}^{*}\right\}_{t=T_{m}^{*}}^{T}\right\}
$$

In the first numerical example of Section 4 we will consider a single structural change as opposed to a sequence of them. In this instance $T_{m}=T_{m}^{*}-1$. In that instance we will often refer to the interval $t=1, \ldots, T_{m}^{*}-1$ as the 'first interval', and $t=T_{m}^{*}, \ldots$ as the 'second interval'. The second of our illustrations in Section 4 deals with two structural changes.

\subsection{Regime Shifts with Beliefs Matching Reality}

As seen in the solution method for models without structural change a key element is to replace the forward expectations with a function that is consistent with the existing model and the information which agents possess. Thus we need to specify both how expectations are to be formed at a point in time and what information is available to agents at that point. In this subsection, agents' beliefs about the prevailing structure are taken to be accurate (i.e. beliefs match reality). We consider two cases. In the first case, the sequence of structural changes given by Equations (7) and (8) is taken to be known once the changes occur. In the second case it is assumed that the sequence of structural changes given by Equations (7) and (8) is foreseen from $T_{m}$. In particular, from period $T_{m}$ onwards, agents know when all future structural changes occur i.e. at the time of the first structural change they know exactly when future changes will take place. ${ }^{3}$

\subsubsection{Structural Changes Known Once They Occur}

To begin, take the simple case of a single structural change. Up until $T_{m}-1=T_{m}^{*}-1$ agents will assume that in the first interval the model with coefficients $\theta=\left\{A_{0}, B_{0, \cdots}\right\}$

\footnotetext{
${ }^{3}$ It will be obvious from the solution method that situations where only some of the future structural changes are known at $T_{m}$ can be readily handled.
} 
is going to continue indefinitely. Hence the solution is just that for the no structural change case i.e. $y_{t}=C+Q y_{t-1}+G \varepsilon_{t}$. From $T_{m}^{*}$ onwards agents form expectations with the final model which has coefficients $\theta^{*}=\left\{A_{0}^{*}, B_{0}^{*}, \ldots\right\}$, and so the solution will be $y_{t}=C^{*}+Q^{*} y_{t-1}+G^{*} \varepsilon_{t}$. Consequently, one simply uses the model that holds at any point $t$ in order to compute the solution for $y_{t}$. Clearly, the solution generalises to any number of unanticipated structural changes.

\subsubsection{Foreseen Structural Changes}

Now consider what happens if, after the first structural change, agents know when all future changes will take place. In this situation expectations need to be formed which recognise that agents know that different structures will hold at some point in the future. In general, from $T_{m}$ onwards the solution for $y_{t}$ at any point in time will be a time varying VAR of the form

$$
y_{t}=C_{t}+Q_{t} y_{t-1}+G_{t} \varepsilon_{t} .
$$

Because the information about future structures (models) is taken to be certain and non-stochastic, it follows that $\mathbb{E}_{t} y_{t+1}=C_{t+1}+Q_{t+1} y_{t}$. Then, following the earlier solution method, we would get the equivalent conditions to Equations (4) to (6) as

$$
\begin{aligned}
\left(I-B_{t} Q_{t+1}\right)^{-1}\left(\Gamma_{t}+B_{t} C_{t+1}\right) & =C_{t} \\
\left(I-B_{t} Q_{t+1}\right)^{-1} A_{t} & =Q_{t} \\
\left(I-B_{t} Q_{t+1}\right)^{-1} D_{t} & =G_{t},
\end{aligned}
$$

where, as before, $\Gamma_{t} \equiv A_{0, t}^{-1} C_{0, t}, A_{t} \equiv A_{0, t}^{-1} A_{1, t}, B_{t} \equiv A_{0, t}^{-1} B_{0, t}$ and $D_{t} \equiv A_{0, t}^{-1} D_{0, t}$. There are two key differences. One is the second condition, which now becomes

$$
A_{t}-Q_{t}+B_{t} Q_{t+1} Q_{t}=0
$$

showing that the solution for $Q_{t}$ involves a backward recursion. To implement this we start from the solution of the final structure $Q_{T_{m}^{*}}=Q^{*}$, and then choose the sequence $\left\{Q_{t}\right\}_{t=T_{m}}^{T_{m}^{*}-1}$ that satisfies Equation (13). As shown in Cagliarini and Kulish (2013) the existence and uniqueness of a solution to the final structure - existence and uniqueness 
of $Q^{*}$ - are necessary conditions for the existence and the uniqueness of the sequence $\left\{Q_{t}\right\}_{t=T_{m}}^{T_{m}^{*}-1}$. Clearly, for a given set of values for the structural parameters of the final model, it will be necessary that they satisfy the Blanchard-Khan conditions. However, because one has to work back to previous models it will also also be necessary that $\left(I-B_{t} Q_{t+1}\right)$ be of full rank, so as to provide a solution for $Q_{t}$ from Equation (11). Cagliarini and Kulish (2013), provide a rank condition that enables one to check for existence and uniqueness of the transition path under any arbitrary finite sequence of structural changes. The second difference comes from the nature of the first condition. This can now be written as

$$
\Lambda_{t}+F_{t} C_{t+1}=C_{t}
$$

where $\Lambda_{t}=\left(I-B_{t} Q_{t+1}\right)^{-1} \Gamma_{t}$ and $F_{t}=\left(I-B_{t} Q_{t+1}\right)^{-1} B_{t}$. With $Q_{t}$ in hand we solve for $C_{t}$ through a forward recursion, yielding $C_{t}=\Lambda_{t}+F_{t} \Lambda_{t+1}+F_{t} F_{t+1} \Lambda_{t+2}+\cdots$. Consequently our algorithm involves both a backward and forward step and we describe it as the BF algorithm. ${ }^{4}$

To illustrate, consider the case of two structural changes. From $T_{m}$ onwards agents know about any future structural changes. Starting with the final interval $T_{m}^{*}, \ldots, T$, because the final model is in place from $T_{m}^{*}$ onwards, one can apply the "no structural change" solution method to get a VAR structure $y_{t}=C^{*}+Q^{*} y_{t-1}+G^{*} \varepsilon_{t}$. Accordingly, this applies to the last interval and enables us to determine that $Q_{T_{m}^{*}}=Q^{*}$. At $t=T_{m}^{*}-1$ the second interval model, which is characterized by coefficients $\bar{\theta}$, is in place, but agents know that the final model holds at $T_{m}^{*}$ onwards, so they account for this when forming expectations. Hence, one solves for $Q_{T_{m}^{*}-1}$ using the backward recursion in Equation (13) but with $A_{t}=\bar{A}$ etc. Before $T_{m}$ the data are generated by the initial model with coefficients $\theta$, that is by the "first interval" VAR structure $y_{t}=C+Q y_{t-1}+G \varepsilon_{t}$.

Hence in the interval, $t=T_{m}, \ldots, T_{m}^{*}-1$, the solution is a time-varying coefficient VAR with the movements in its coefficients being pinned down by the way the structure changes and is expected to change. Notice that the backward recursion implied by Equation (13) makes $Q_{t}$ a function of $Q_{t+1}$. This means that the weights use to form expectations at

\footnotetext{
${ }^{4}$ To simulate the model under structural change when break dates are known then these may be imposed and shocks may be drawn and put through the solution. If date breaks were estimated, these may be sampled from the estimated posterior distribution. $M$ samples of $T$ periods conditioned on the structural changes may be constructed to form averages and compute moments.
} 
time $t$ are a function of current and future structures (models). ${ }^{5}$

\subsubsection{Announcement Effects}

Preannouncement effects, such as happens with the introduction of a Goods and Services Tax (GST), the formation of a common currency, etc. can be captured in the setup above. In this instance the origin of beliefs is clear - it is the announcement of a change in the parameters. The only question arising is when agents learn about the change? If there is a single regime shift which is known in advance of when it occurs, then the initial model would hold for $t=1, \ldots, T_{m}^{*}-1$ and the final model from $t=T_{m}^{*}, \ldots, T$. The date of the break, $T_{m}^{*}$, is the time when the final model is in place. However agents may only learn about the forthcoming change at, say, $T_{a}$. As before, we would choose the sequence $\left\{Q_{t}\right\}_{t=T_{a}}^{T_{m}^{*}-1}$ starting from $Q_{T_{m}^{*}}=Q^{*}$ such that $A-Q_{t}+B Q_{t+1} Q_{t}=0$. Then for $t=T_{a}, \ldots, T_{m}^{*}-1$, the structure remains constant (i.e $A_{0, t}=A_{0}, C_{0, t}=C_{0}$, etc.), and so the announcement itself triggers a drift in the reduced form. In fact, between the announcement date, $T_{a}$, and the implementation date, $T_{m}=T_{m}^{*}$, the reduced form drifts from the "first interval" VAR structure $y_{t}=C+Q y_{t-1}+G \varepsilon_{t}$ towards the "final interval" VAR structure, $y_{t}=C^{*}+Q^{*} y_{t-1}+G^{*} \varepsilon_{t}$.

\subsection{Regime Shifts where Beliefs are Different from Reality}

In the analysis above beliefs agree with reality. When the structural changes are unknown until they occur, expectations are formed at each point in time using the model that pertains to that period of time. When agents foresee the structural changes, and the structural changes do take place, they know both the new and old models, and therefore form expectations by weighting the information appropriately at each point in time. In this section we deal with the more general case in which this may not always be true. In doing so we assume agents do eventually use the correct model but there may be a period

\footnotetext{
${ }^{5}$ Cúrdia and Finocchiaro (2005) estimate a model for Sweden which has a change in the interest rate rule at a known point in time. They estimate the model of the first monetary regime by assuming that in the period before the break date agents expect that this will continue indefinitely and, once the break happens, there will be a new monetary regime. They then estimate both models separately. Estimation of the model before the break-date is standard but to estimate the model of the second regime they need to assume some initial values for the Kalman filter after the break date. The backward-forward algorithm does not estimate the models separately and so does not need to make any assumption about the values of the Kalman filtered variables around the break-date.
} 
of time in which they are mistaken about which structure (model) holds. Hence, during that interval, they may form incorrect expectations: expectations are model consistent, but consistency may be with the wrong model for part of the sample period.

We introduce notation for the timing of beliefs. We denote by $T_{b}$ the time when agents update their beliefs about current and future structures and by $T_{b}^{*}$ the time when beliefs agree with the final structure. We impose no restrictions between $T_{m}$ and $T_{m}^{*}$ on the one hand and $T_{b}$ and $T_{b}^{*}$ on the other, so that beliefs may converge either before or after the structure has converged, and they may be updated either before or after the first structural change.

One possibility is illustrated in Figure 3. The lower arrows describe, as before, the evolution of the structure while the upper arrows now describe the evolution of beliefs. The sequence of structural changes begins in $T_{m}$ and ends in $T_{m}^{*}$, with beliefs being based on the wrong structure (model) for some time. Beliefs are first updated in period $T_{b}$, after the structural changes begin, and converge in period $T_{b}^{*}$, after the structure has converged.

This generalisation allows us to consider situations in which agents do not get the timing of the structural changes right, as well as capturing situations of imperfect credibility in which policy announcements may be carried out as announced, but are not necessarily fully incorporated into expectations formation.

We assume the structure evolves as before: that is, before $T_{m}$ the structure is stable at Equation (1). Then, during $t=T_{m}, \ldots, T_{m}^{*}-1$, the structure evolves as in Equation (7), subsequently changing for $t \geq T_{m}^{*}$ to Equation (8). Agents' beliefs, however, may evolve differently. Before $T_{b}$ expectations are based on Equation (1) while after $T_{b}$ agents believe that the structural coefficients will evolve as follows:

$$
\left\{\begin{array}{lllll}
\tilde{A}_{0, t} & \tilde{C}_{0, t} & \tilde{A}_{1, t} & \tilde{B}_{0, t} & \tilde{D}_{0, t}
\end{array}\right\}_{t=T_{b}}^{T_{b}^{*}-1} .
$$

Subsequently, beliefs change for $t=T_{b}^{*}, \ldots, T$ to Equation (8), the final structure. Equation (14) indicates that, in the period up to $T_{b}^{*}$, agents may have inaccurate beliefs about which model is generating the data. In the special case that $A_{0, t}=\tilde{A}_{0, t}$ etc., $T_{m}=T_{b}$ and $T_{m}^{*}=T_{b}^{*}$, so that beliefs are always accurate and the situation coincides with the one discussed in Section 3.1.2. 
In terms of our single structural change example, the period up to $T_{b}^{*}$ may have a period of time over which the initial model holds and a further period for which the final model holds. From $\max \left(T_{b}^{*}, T_{m}^{*}\right)$ onwards it is only the final model that generates the data.

Given this departure from the standard rational expectations context, it is assumed that agents combine observed outcomes with their beliefs about the structure when computing the time $t$ conditional expectation, $\tilde{\mathbb{E}}_{t} y_{t+1}$, where the notation emphasises that expectations are based on the model with parameters in Equation (14). ${ }^{6}$ In this case, agents use their model beliefs to determine weights to be applied to observed data when forming expectations. When agents believe the structure will evolve as captured by Equation (14), one proceeds as before, starting from $\tilde{Q}_{T_{b}^{*}}=Q^{*}$ to find the sequence $\left\{\tilde{Q}_{t}\right\}_{t=T_{b}}^{T_{b}^{*}-1}$ such that

$$
\tilde{A}_{t}-\tilde{Q}_{t}+\tilde{B}_{t} \tilde{Q}_{t+1} \tilde{Q}_{t}=0
$$

The solution agents would infer for $t=T_{b}, \ldots, T_{b}^{*}-1$ is

$$
y_{t}=\tilde{C}_{t}+\tilde{Q}_{t} y_{t-1}+\tilde{G}_{t} \tilde{\varepsilon}_{t},
$$

which implies that $\tilde{\mathbb{E}}_{t} y_{t+1}=\tilde{C}_{t+1}+\tilde{Q}_{t+1} y_{t}$. However, the actual path of the economy obeys

$$
A_{0, t} y_{t}=C_{0, t}+A_{1, t} y_{t-1}+B_{0, t} \tilde{\mathbb{E}}_{t} y_{t+1}+D_{0, t} \varepsilon_{t} .
$$

Using Equation (16) it is easy to show that the reduced-form VAR which is the solution to (17) is given by

$$
y_{t}=\hat{C}_{t}+\hat{Q}_{t} y_{t-1}+\hat{G}_{t} \varepsilon_{t}
$$

\footnotetext{
${ }^{6}$ One could alternatively assume that agents utilise their beliefs about the model to produce both the weights and values for the endogenous variables themselves when computing expectations i.e. they project a model consistent path for the endogenous variables which will be incorrect if model beliefs are incorrect. There are other reasonable assumptions as well. For example, we could assume that either only lagged outcomes are observed or that only some subset of the variables are observed at time $t$. These extensions are left for further research.
} 
where

$$
\begin{aligned}
& \hat{C}_{t}=\left(A_{0, t}-B_{0, t} \tilde{Q}_{t+1}\right)^{-1}\left(C_{0, t}+B_{0, t} \tilde{C}_{t+1}\right), \\
& \hat{Q}_{t}=\left(A_{0, t}-B_{0, t} \tilde{Q}_{t+1}\right)^{-1} A_{1, t}, \\
& \hat{G}_{t}=\left(A_{0, t}-B_{0, t} \tilde{Q}_{t+1}\right)^{-1} D_{0, t} .
\end{aligned}
$$

The solution in this case also takes the form of a time-varying coefficient VAR with movements in its coefficients being pinned down by the way the structure evolves, as well as agents' beliefs about these structural changes.

When the structural changes begin before agents first update their beliefs (i.e. $T_{m}<$ $T_{b}$ ) - as is the case in Figure 3 - expectations are based on the initial structure in those periods, that is $\tilde{\mathbb{E}}_{t} y_{t+1}=C+Q y_{t}$, so the economy evolves as

$$
A_{0, t} y_{t}=C_{0, t}+A_{1, t} y_{t-1}+B_{0, t}\left(C+Q y_{t}\right)+D_{0, t} \varepsilon_{t},
$$

during those periods. With $\tilde{\mathbb{E}}_{t} y_{t+1}=\tilde{C}_{t+1}+\tilde{Q}_{t+1} y_{t}$ in hand, other cases, $T_{m}>T_{b}$ or $T_{m}^{*}>T_{b}^{*}$, are straightforward to compute.

\subsection{The Likelihood}

As we have discussed above, a set of structural changes and assumptions about beliefs and expectations formation map into a sequence of reduced-form matrices. If the structural changes are unknown until they occur, the solution is computed as in Section 3.1.1. If the structural changes are foreseen the system follows Equation (9), and, in the more general formulation where beliefs may differ from reality, the system follows Equation (18). The derivation of the likelihood is identical in each case, involving a sequence of reduced form matrices. Therefore, with no loss of generality, let the reduced form be given by Equation $(9)$ :

$$
y_{t}=C_{t}+Q_{t} y_{t-1}+G_{t} \varepsilon_{t} .
$$


Now assume that we have in hand a sample of data, $\left\{z_{t}\right\}_{t=1}^{T}$, where $z_{t}$ is a $n_{z} \times 1$ vector of observable variables that relate to the model's variables through

$$
z_{t}=H y_{t}+v_{t}
$$

In Equation (19), $v_{t}$ is an iid measurement error with $\mathbb{E}\left(v_{t}\right)=0$ and $\mathbb{E}\left(v_{t} v_{t}^{\prime}\right)=V$. The observation equation, Equation (19), and the state equation, Equation (9), constitute a state space model. Therefore, the Kalman filter can be used to construct the likelihood function for the sample $\left\{z_{t}\right\}_{t=1}^{T}$, as originally proposed by Schweppe (1965) and outlined, for example, in Harvey (1989). The Appendix provides details of the derivation of the log-likelihood in Equation (20).

$$
\mathcal{L}=-\left(\frac{n_{z} T}{2}\right) \ln (2 \pi)-\frac{1}{2} \sum_{t=1}^{T} \ln \operatorname{det}\left(H \Sigma_{t \mid t-1} H^{\prime}+V\right)-\frac{1}{2} \sum_{t=1}^{T} u_{t}^{\prime}\left(H \Sigma_{t \mid t-1} H^{\prime}+V\right)^{-1} u_{t}
$$

In Equation $(20), u_{t}=z_{t}-\mathbb{E}_{t-1} z_{t}$ is the prediction error, $\Sigma_{t \mid t-1}=\mathbb{E}_{t-1}\left(\left[y_{t}-\mathbb{E}_{t-1} y_{t}\right]\left[y_{t}-\right.\right.$ $\left.\left.\mathbb{E}_{t-1} y_{t}\right]^{\prime}\right)$ is the covariance matrix of the state variables $y_{t}$ conditional on information at $t-1$, and $\operatorname{cov}_{t-1}\left(z_{t}\right)=H \Sigma_{t \mid t-1} H^{\prime}+V$.

With Equation (20) in hand, standard likelihood-based tests for parameter stability and detection of break-dates are available. ${ }^{7}$ This is a key product of our approach. Although in the formulation we treat the dates at which new structures emerge as known by agents, it is possible for the econometrician to find an estimate of these dates from the likelihood values i.e. to determine when the structural changes actually took place. Of course changes in the likelihood are the basis for many tests of structural change e.g. Andrews (1993) and Bai and Perron (1998).

\footnotetext{
${ }^{7}$ Under the null hypothesis of no structural change the likelihood ratio statistic, $2\left(\mathcal{L}\left(\hat{\theta}_{U}\right)-\mathcal{L}\left(\hat{\theta}_{R}\right)\right)$, is asymptotically distributed as a chi-square random variable with $m=\operatorname{dim}\left(\hat{\theta}_{U}\right)-\operatorname{dim}\left(\hat{\theta}_{R}\right) \operatorname{degrees}$ of freedom, where $\hat{\theta}_{U}$ is the unrestricted maximum likelihood estimate of the vector of structural parameters while $\hat{\theta}_{R}$ is the restricted maximum likelihood estimate of the vector of structural parameters after imposing the restrictions of no structural change. Detection of structural change is generally done with a recursive likelihood ratio test.
} 


\section{Applications}

In this section we look at two applications of the BF algorithm for dealing with structural change. In the first an examination is made of the consequences of announcing an increased inflation target under situations where the announced change is credible and when it is not. The second application is an empirical one which looks at the time taken for beliefs about the new (implicit) inflation target implemented under the Volcker regime to coincide with reality. In both cases we will utilize the quarterly New Keynesian model of Ireland (2004) described below in Equations (21)-(27). ${ }^{8}$

$$
\begin{aligned}
x_{t} & =(\bar{r}-\bar{\pi})-\left(r_{t}-\mathbb{E}_{t} \pi_{t+1}\right)+\mathbb{E}_{t} x_{t+1}+(1-\omega)\left(1-\rho_{a}\right) a_{t} \\
\pi_{t} & =\bar{\pi}+\beta\left(\mathbb{E}_{t} \pi_{t+1}-\bar{\pi}\right)+\psi x_{t}-e_{t} \\
r_{t} & =\bar{r}+\rho_{r}\left(r_{t-1}-\bar{r}\right)+\rho_{\pi}\left(\pi_{t}-\bar{\pi}\right)+\rho_{g}\left(g_{t}-\bar{g}\right)+\rho_{x} x_{t}+\varepsilon_{r, t} \\
x_{t} & =\hat{y}_{t}-\omega a_{t} \\
g_{t} & =\bar{g}+\hat{y}_{t}-\hat{y}_{t-1}+\varepsilon_{z, t} \\
a_{t} & =\rho_{a} a_{t-1}+\varepsilon_{a, t} \\
e_{t} & =\rho_{e} e_{t-1}+\varepsilon_{e, t}
\end{aligned}
$$

In the equations above, $x_{t}$ is the output gap, defined as the deviation of output from a socially efficient level of output; $\pi_{t}$ is the gross rate of inflation, that is $\ln \left(p_{t} / p_{t-1}\right) ; r_{t}$ is the $\log$ of the gross nominal interest rate; $g_{t}$ is the growth rate of output and $\hat{y}_{t}$ is the percentage deviation of output, $Y_{t}$, from $Z_{t}$, the level of total factor productivity. The $\log$ of $Z_{t}$ follows a unit root with drift $\bar{g}$. Finally, $a_{t}$ is a demand shock, and $e_{t}$ is a cost-push shock. The $\varepsilon$ 's are identically and independently distributed shocks. In steady state, $\pi_{t}=\bar{\pi}, r_{t}=\bar{r}, g_{t}=\bar{g}$ and $\bar{r}=\bar{\pi}+\bar{g}-\ln \beta$; all other variables (including the output gap) settle on zero. The reason we choose to leave some variables in levels, as opposed to percentage deviations from steady state, is that there are changes in the steady states of these variables in the examples that follow.

\footnotetext{
${ }^{8} \mathrm{~A}$ previous version of this paper contains additional estimation exercises on simulated data. To preserve space we refer the reader to Kulish and Pagan (2012). Our choice of simulated data is to be able to test the procedure and to assess the precision of the estimates of those parameters which only appear in sub-samples of the data.
} 


\subsection{Lifting the Inflation Target}

Recently, the Bank of Japan announced its intentions to achieve an annual inflation target of 2 percent in two years. In the May 2013 minutes, some members of the Bank of Japan's board have indeed noted that: "[...] it seemed difficult to achieve around 2 percent inflation towards the latter half of the projection period, since it was highly uncertain whether inflation expectations would lead to a rise in the actual rate of inflation [...]" This suggests that the success of the policy depends crucially on its credibility through an impact on inflation expectations, and we therefore examine that qualification through an experiment.

As Table 1 shows the inflation target starts at $\bar{\pi}^{(1)}=.00125$ and is lifted over three years to $\bar{\pi}^{(2)}=.005$. It grows from one position to the other at a constant rate, so that between $T_{m}=4$ and $T_{m}^{*}=16$ the target is not fixed but has values $\bar{\pi}_{t}$. Thus, as foreshadowed in our earlier theoretical discussion, there are twelve structures operating between $T_{m}$ and $T$. At the parameter values of Table 1, impulse responses are compared for two cases: firstly where the announced plan to lift the inflation target is credibly anticipated by markets and carried out as announced and, secondly, where there is no credibility. A lack of credibility means that agents' beliefs are that the target remains at $\bar{\pi}^{(1)}$ i.e. agents' beliefs remain anchored at the old target.

Figure 4 shows impulse responses for the nominal interest rate, inflation and the output gap to a contractionary demand shock. Up until the fourth period the economy has a low steady-state inflation rate. The demand shock is applied in the fourth period, at which point the central bank announces its changed policy, beginning to gradually lift the inflation target from 0.5 to 2 percent over the next twelve quarters. This is shown in the bottom right-hand panel of figure 4. Figure 4 also shows conventional impulse responses under the high and low inflation regimes. As one would expect, in the absence of a policy that increases the inflation target, a contractionary demand shock initially lowers inflation and the output gap which, through the operation of a Taylor rule, leads to lower nominal interest rates.

When the announcement is credible and agents anticipate future increases of the inflation target, inflation and inflation expectations rise on impact. The policy, when credible, 
is powerful enough to decrease the real interest rate and offset the deflationary impact that the shock would have otherwise had. Thereafter, within a few quarters, all of inflation, the interest rate and output are already aligned to the responses that would have prevailed in the 2 percent inflation regime. The situation is quite different when expectations are anchored at the lower target: inflation expectations and inflation fall initially and only start to increase gradually as the central bank cuts the nominal interest rate to offset deviations from an increasing target. Even two years after it has stopped increasing the target inflation rate it is evident that inflation remains well below the higher target of $\bar{\pi}^{(2)}$, which agrees with the caution sounded by the Bank of Japan's Board.

\subsection{The Volcker Disinflation}

Next, we consider an episode which occurred during the Volcker disinflation. It is known from expectations data that agents did not immediately adapt their beliefs to what has been characterized as a shift in inflation targets during Chairman Volcker's time at the Federal Reserve. In order to examine this we extend the model given by Equations (21)(27). Firstly, we replace the policy rule, Equation (23), with

$$
r_{t}=\bar{r}_{t}+\rho_{r}\left(r_{t-1}-r\right)+\rho_{\pi, t}\left(\pi_{t}-\bar{\pi}_{t}\right)+\rho_{g, t}\left(g_{t}-\bar{g}\right)+\varepsilon_{r, t}
$$

which allows for time-variation in the inflation target, $\bar{\pi}_{t}$. Before $1979 \mathrm{q} 4$ we characterize the target as evolving in the following way

$$
\bar{\pi}_{t}=\left(1-\rho^{*}\right) \bar{\pi}^{(1)}+\rho^{*} \bar{\pi}_{t-1}+\varepsilon_{\pi, t}^{*},
$$

where $\pi^{(1)}$ might be regarded as a target rate inherited from the 1960s and 1970s ( perhaps the unconditional mean of inflation) and $\varepsilon_{\pi, t}^{*}$ is an nid shock with zero mean and standard deviation $\sigma_{\pi^{*}}$. The parameters $\rho_{\pi}$ and $\rho_{g}$ are fixed at values of $\rho_{\pi}^{(1)}$ and $\rho_{g}^{(1)}$ up until $1979 \mathrm{q} 4$ but then change to $\rho_{\pi}^{(2)}$ and $\rho_{g}^{(2)}$ (these are estimated and it may be that the values are the same). After 1979q4 the target evolves as

$$
\bar{\pi}_{t}=\left(1-\rho^{*}\right) \bar{\pi}^{(2)}+\rho^{*} \bar{\pi}_{t-1} .
$$


Hence the target eventually becomes $\bar{\pi}^{(2)}$. The date of the change in the target is not anticipated. As well as structural breaks in the inflation target setting we need to make an allowance for the fact that the standard deviations of shocks declined over this period of time, as it included what became known as the Great Moderation. To this end we assume that the date $T_{\sigma}$ is when standard deviations change. This will be estimated. To do so we use a parsimonious specification and introduce the parameter $\gamma$, which multiplies all standard deviations before $T_{\sigma}$ i.e. we assume that the standard deviations of all variables shift in the same proportions. Both $\gamma$ and $T_{\sigma}$ are then estimated. It is assumed that the beginning of the Volcker disinflation is at $1979 q 4$, the quarter when the Federal Reserve made its important announcement of new operating procedures (see Goodfriend and King (2005)). Beliefs can follow the pre-Volcker regime for some period of time after 1979q4. The date when beliefs re-align with reality, $T_{b}$, has therefore to be estimated. As we let $T_{b} \geq 1979 \mathrm{q} 4$, beliefs can possibly shift at the same time as the final inflation target shifts.

Estimation of the model uses data on core inflation, real per capita GDP growth, the Federal Funds rate and the Michigan measure of inflation expectations. ${ }^{9}$ The sample runs from $1957 \mathrm{q} 2$ to $2008 \mathrm{q} 1 .^{10}$ Because the series for the Michigan measure of inflation expectations begins in 1978q1, we adapt the Kalman filter to handle a time-varying number of observables. The data are shown in the on-line appendix.

We add an observation equation for the Michigan measure of inflation expectations of the form

$$
\mathbb{E}_{t}^{o b s} \pi_{t+1}=\frac{1}{4}\left(\sum_{j=1}^{4} \mathbb{E}_{t} \pi_{t+j}\right)+\eta_{t}
$$

where $\eta_{t}$ is a measurement error that follows an $\operatorname{AR}(1)$

$$
\eta_{t}=\rho_{\eta} \eta_{t_{1}}+\varepsilon_{\eta, t}
$$

and $\varepsilon_{\eta, t}$ is an nid shock with zero mean and standard deviation $\sigma_{\eta}$.

\footnotetext{
${ }^{9}$ We choose the Michigan Survey measure of inflation expectations which starts in 1978q1 before the Volcker disinflation begins, instead of the also popular measure from the Survey of Professional Forecasters which starts in 1981q3.

${ }^{10}$ Our methodology can handle estimation at the zero lower bound as shown in Kulish et al. (2014), but, for simplicity, our sample ends before the Federal Funds rate reaches the zero lower bound. This seems appropriate for the questions we are looking at.
} 
As is common in the DGSE estimation literature we put priors on the structural parameters, $p(\theta)$, a uniform prior on the dates, $p\left(T_{b}, T_{\sigma}\right)$ and use Markov Chain Monte Carlo methods to approximate the posterior density $p\left(\theta, T_{b}, T_{\sigma} \mid Z\right) \propto \mathcal{L}\left(Z \mid \theta, T_{b}, T_{\sigma}\right) p(\theta) p\left(T_{b}, T_{\sigma}\right)$ where $Z \equiv\left\{z_{t}\right\}_{t=1}^{T}$ is the data. We construct a chain of 575,000 draws from this joint density and throw out the first 25 per cent as the burn-in sample. ${ }^{11}$

We find a 30 per cent chance that beliefs shifted in the quarter that the Volcker disinflation began, and there are significant spikes in probability of 20 per cent in the 1980q1 and 1980q4 quarters. ${ }^{12}$ There is a 27 per cent chance that beliefs shifted after 1981, but no particular quarter appears more likely. The date when the variance of shocks fell is estimated to have taken place between 1983q1 and 1985q1.

Inflation expectations data are particularly helpful in identifying $T_{b}$. In fact, we have re-estimated the model without inflation expectations data and, while we find a similar cumulative distribution for $T_{\sigma}$, it is the case that more mass is shifted towards later dates for $T_{b}$. Specifically, when expectations data are not used, there is a 30 per cent probability that beliefs shifted in the quarter that the Volcker disinflation began, while the remaining mass is distributed fairly evenly throughout the 1980's and early 1990's.

The estimates of the associated structural parameters are shown in Table 2. We find, like Sims and Zha (2006) and Primiceri (2005), a significant fall in the variance of structural shocks in the United States. Our estimated mode for $\gamma$, the parameter that multiplies standard deviations before $T_{\sigma}$, is 2.25 . This means that the size of shocks have more than halved since the early 1980's. We also find differences in policy parameters, in particular, in that for the long-run rate of inflation, which is lower and more precisely estimated in the post-Volcker regime.

\section{Relation to the Markov-switching Approach}

A number of authors have proposed handling rational expectations models with structural change using switches between regimes. The date of switches are exogenously generated

\footnotetext{
${ }^{11}$ We set up the posterior sampler in two blocks as described in the on-line appendix, one for the time of breaks (referred to as break-dates) and one for structural parameters. See also Kulish et al. (2014).

${ }^{12}$ See the on-line appendix for a plot of the cumulative distributions for the date breaks.
} 
via a Markov-switching (MS) process that is characterized by a given set of transition probabilities (see Cho (2015), Davig and Leeper (2007), Farmer et al. (2009) and Farmer et al. (2011), and Bianchi (2012)). The transition probabilities are known to agents in the model but unknown to the econometrician. The number of regimes is generally kept quite small. As mentioned in the introduction, each time there is a switch from one regime to another there will be a structural break.

It is worth considering some of the conceptual and practical differences between our approach and that used by MS modellers. This was done by working with a three equation New Keynesian (NK) model comprising inflation, an output gap and a nominal interest rate. A single structural break in the response of interest rates to inflation was then generated via a two regime MS model. Data was simulated from such a model and both methods for handling the structural break were compared. The details of the experiment and results are in section 4 of the on-line appendix.

Both approaches produce good estimates of the break dates as well as the magnitude of the shift in the value of the structural parameter, although the BF method seemed to be marginally better. Moreover, it took three times as long to produce the results from the MS algorithm as compared to the $\mathrm{BF}$ algorithm. This suggests a computational advantage to our method. It might be thought that the MS approach could be superior when there are many break dates, although it is unclear why we would expect many shifts in parameters. To investigate this we used the same NK model but now with three structural changes in the single parameter. The BF method still worked better and was faster to apply than the MS approach. In fact, computational time for it was largely unaffected from when there was just a single structural change. It should be observed that the experiment we perform has been designed to favour the MS approach in that structural change came from a Markov switching process.

Section 4 of the on-line appendix also discusses some cases where it would be easier to use the BF method. One of these is when the break dates are known and not the result of some stochastically exogenous process. In that instance the MS approach would have to deal with absorbing states. Another is when there are many parameters undergoing structural change but not at the same time i.e. there are many regimes. It is well known that MS models have estimation difficulties in this context - one reason being that the algo- 
rithms to compute likelihoods (or posteriors) are quite complex, numerically challenging, and not assured of either finding a maximum to the likelihood or of avoiding multi-modal posteriors. ${ }^{13}$ Indeed, it has often been the case that MS researchers have had to assume that the breaks occur in all the parameters at the same time in order to avoid estimation problems. An issue in the MS approach is that it necessary to work with an approximation to the likelihood, since the number of states must be collapsed in order to make the Kalman filter operable. This is explained in Kim and Nelson (1999). Even with this approximation, the number of operations required by the Kim-Nelson filter is proportional to the square of the number of states. MS estimation also requires the additional steps of the Hamilton filter. In contrast the on-line appendix shows that, in implementing the BF algorithm, the sampler uses two blocks, one for the structural parameters and one for the break-dates. This requires only one additional evaluation of the likelihood. Consequently, our method leads to a straightforward computation of the likelihood, and this is likely to be particularly useful in larger models with many breaks in parameters. More discussion of the relative utility of each approach is given in section 4 of the on-line appendix. Each has advantages and disadvantages which are context-dependent.

\section{Conclusion}

In this paper we develop a solution for linear models in which agents use model-consistent expectations but there is structural change over time. The solution allows for agents' beliefs about the structure to depart from the correct model of the economy, at least for a period of time. In all cases the solution takes the form of a time-varying coefficient VAR. This can be put into a state space form and the Kalman filter can then be used to construct the likelihood enabling estimation of the parameters and dates of structural change to be done by classical or Bayesian methods. The solution method is transparent and it is shown in the paper's online appendix that it works well when compared to a Markov-Switching alternative. The fact that it is a relatively simple extension of the standard methods used for solving linear models with forward-looking expectations should make it attractive both

\footnotetext{
${ }^{13}$ See p.17 in Liu et al. (2011) and Baele et al. (2015) who acknowledge that estimation of a stylized MS rational expectations model is a very complex task. Moreover, as emphasised by Cho (2015), the necessary conditions for determinacy are not yet known in a class of general MS models.
} 
for empirical work and for understanding issues relating to the existence of solutions.

Not all issues were solved in this paper. In the theoretical and empirical work we proceeded under the assumption that agents' beliefs either agree with reality or that the period of departure is for a fixed period of time. This period can be estimated by the econometrician. Hence, we abstract from describing exactly how the agents come to know of the structural change. In the current formulation, agents use the old model parameters for a period in time (which might be zero periods) after the shift has actually taken place before they become aware of the new values. What makes them aware of these new values is not fully described. In some instances, it could come from announcements, as with a changed inflation target or new tax rates. No learning is needed in those cases. Moreover, it is not entirely clear how agents actually do learn in practice about new parameter values. The standard learning specifications that postulate recursive least squares algorithms are as much of an assumption as the one that we employ - namely that there is a point in time when agents come to know the new parameter values. If one wanted agents to learn about the parameters (rather than eventually knowing them as in our solution), it would be necessary to append a learning specification to the models we work with.

We believe that the learning aspect is best left to another study, as it is of primary importance to understand solution and estimation in the simpler context without a particular specification of learning by agents. ${ }^{14}$ This is especially so given the forward looking nature of the models that we deal with. The key to the BF solution was that we could replace $\mathbb{E}_{t}\left(Q_{t+1} y_{t}\right)$ by $Q_{t+1} y_{t}$, where $Q_{t+1}$ was the time-varying $\operatorname{VAR}(1)$ coefficient matrix that came from the backward recursion. If $Q_{t+1}$ can be taken to be a constant or determined exogenously (as with an announcement) then $\mathbb{E}_{t}\left(Q_{t+1} y_{t}\right)$ can be replaced by $Q_{t+1} y_{t}$ and our algorithm applies directly. If, however, data are used to determine $\mathbb{E}_{t} Q_{t+1}$ then one may end up with a non-linearity in the VAR that accounts for the structural change, and so the time-varying VAR system we deal with here would need to be extended. Because the mapping of $\mathbb{E}_{t} Q_{t+1}$ into data involves many choices about how agents would learn about structural changes, we leave it to future research. The fundamental idea that anticipated structural change involves some form of backward-forward iteration, however, is likely to be present in any generalization. As an aside we observe here that, in general,

\footnotetext{
${ }^{14}$ See Gibbs and Kulish (2015) for an extension along these lines.
} 
none of the alternative MS approaches deal with learning either, so this is clearly a topic that needs further research with all methods.

The models we work with are linear. Recently, Canova et al. (2015) study the econometric implications of using time-invariant models when the true model has time-varying parameters. They solve the latter models using higher order perturbation methods around the non-stochastic steady state of the time-varying parameter model. It would seem possible to replace our linear model with such a representation if what is involved are just changing "slope" coefficients. But in the applications of Section 4 there were changes in the steady state, so that one cannot immediately use the idea of a perturbation around one steady state solution - instead it would have to be around a time varying solution. A BF type algorithm could be deduced to handle such non-linearity, although the notation will be quite complex. Nevertheless, exploring higher order solutions in the presence of changes in the steady state is a worthwhile avenue left for future research.

\section{References}

Anderson GS. (1997). A reliable and computationally efficient algorithm for imposing the saddle point property in dynamic models. Federal Reserve Board, Occasional Staffs Studies 4.

Andrews DW. (1993). Tests for parameter instability and structural change with unknown change point. Econometrica 821-856.

Baele L, Bekaert G, Cho S, Inghelbrecht K, Moreno A. (2015). Macroeconomic regimes. Journal of Monetary Economics 70: 51-71.

Bai J, Perron P. (1998). Estimating and testing linear models with multiple structural changes. Econometrica 47-78.

Bianchi F. (2012). Evolving monetary-fiscal policy mix in the United States. American Economic Review: Papers and Proceedings 102: 167-172.

Binder M, Pesaran MH. (1995). Multivariate Rational Expectations Models and Macroeconometric Modelling: A Review and Some New Results. In Pesaran MH, Wickens 
M, eds., Handbook of Applied Econometrics: Macroeconomics, 139-187. Oxford: Basil Blackwell.

Binder M, Pesaran MH. (1997). Multivariate linear rational expectations models: Characterization of the nature of the solutions and their fully recursive computation. Econometric Theory 13: 877-888.

Blanchard OJ, Kahn CM. (1980). The solution of linear difference models under rational expectations. Econometrica 48: 1305-1311.

Cagliarini A, Kulish M. (2013). Solving linear rational expectations models with predictable structural changes. The Review of Economics and Statistics 95(1): 328-336.

Canova F, Ferrini F, Matthes C. (2015). Approximating time varying structural models with time invariant structures .

Cho S. (2015). Sufficient conditions for determinacy in a class of Markov-switching rational expectations models. Review of Economic Dynamics forthcoming.

Clarida R, Gali J, Gertler M. (2000). Monetary policy rules and macroeconomic stability: Evidence and some theory. Quarterly Journal of Economics 1: 147-180.

Cúrdia V, Finocchiaro D. (2005). An estimated DSGE model for sweden with a monetary regime change. Stockholm University, Institute for International Economic Studies Seminar Papers 740.

Davig T, Leeper EM. (2007). Generalizing the taylor principle. American Economic Review 97: 607-635.

Farmer R, Waggoner D, Zha T. (2009). Understanding markov-switching rational expectations models. Journal of Economic Theory 144: 1849-1867.

Farmer R, Waggoner D, Zha T. (2011). Minimal state variable solutions to markovswitching rational expectations models. Journal of Economic Dynamics and Control 35: $2150-2166$.

Gibbs CG, Kulish M. (2015). Disinflations in a model of imperfectly anchored expectations. CAMA Working Paper 36-2015 . 
Goodfriend M, King RG. (2005). The incredible Volcker disinflation. Journal of Monetary Economics 52: $981-1015$.

Harvey AC. (1989). Forecasting, structural time series models and the Kalman filter. Cambridge: Cambridge University Press.

Inoue A, Rossi B. (2011). Identifying the sources of instabilities in macroeconomic fluctuations. Review of Economics and Statistics 93(4): 1186-1204.

Ireland P. (2001). Sticky-price models of the business cycle: Specification and stability. Journal of Monetary Economics 47: 3-18.

Ireland P. (2004). Technology shocks in the New Keynesian model. The Review of Economics and Statistics 86: 923-936.

Kim CJ, Nelson CR. (1999). State-space models with regime switching: Classical and Gibbs-sampling approaches with applications, volume 2. MIT press Cambridge.

King R, Watson M. (1998). The solution of singular linear difference systems under rational expectations. International Economic Review 39: 1015-1026.

Klein P. (2000). Using the generalized Schur form to solve a multivariate linear rational expectations model. Journal of Economic Dynamics and Control 24: 1305-1311.

Kulish M, Morley J, Robinson T. (2014). Estimating DSGE models with forward guidance. Research Paper 201, UNSW Australia Business School Research Paper.

Kulish M, Pagan A. (2012). Estimation and solution of models with expectations and structural changes. Research Discussion Paper 2012-08, Reserve Bank of Australia.

Liu Z, Waggoner DF, Zha T. (2011). Sources of macroeconomic fluctuations: A regimeswitching DSGE approach. Quantitative Economics 2: 251-301.

Lubik T, Schorfheide F. (2004). Testing for indeterminacy: An application to U.S. monetary policy. American Economic Review 94(1): 190-217.

Primiceri G. (2005). Time varying structural vector autoregressions and monetary policy. Review of Economic Studies 72: 821-852. 
Schweppe FC. (1965). Evaluation of likelihood functions for Gaussian signals. Information Theory, IEEE Transactions on 11: 61-70.

Sims CA. (2001). Solving linear rational expectations models. Computational Economics 20: $1-20$.

Sims CA, Zha T. (2006). Were there regime switches in U.S. monetary policy? The American Economic Review 54-81.

Stock JH, Watson MW. (2007). Why has U.S. inflation become harder to forecast? Journal of Money, Credit and Banking 39: 3-33.

Uhlig H. (1999). A toolkit for analyzing nonlinear dynamic stochastic models easily. In Marimon R, Scott A, eds., Computational Methods for the Study of Dynamic Economies, 30-61. Oxford University Press. 


\section{List of Figures}

1 Inflation and inflation targets in Chile ${ }^{*} \ldots \ldots \ldots 30$

2 Timing of Structural Changes . . . . . . . . . . . . . . . . . . . 31

3 Timing of Structural Changes and Beliefs . . . . . . . . . . . . . . . 32

4 Impulse responses to a contractionary demand shock . . . . . . . . . . . . . 33 
Figure 1: Inflation and inflation targets in Chile *

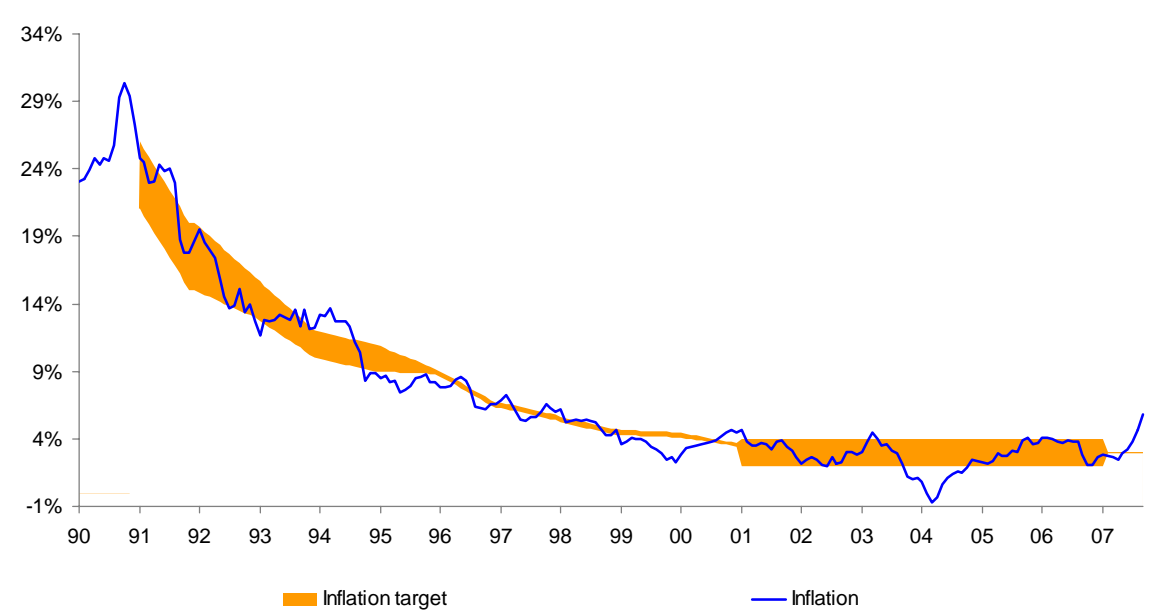

* Notes: 1990-99: Annual targets announced every September for next years December/December inflation. In September 1999, the central bank announced a point target for 2000 and also, starting in 2001, a stationary target range for the indefinite future. Since 2007, the target is 3 per cent. 
Figure 2: Timing of Structural Changes

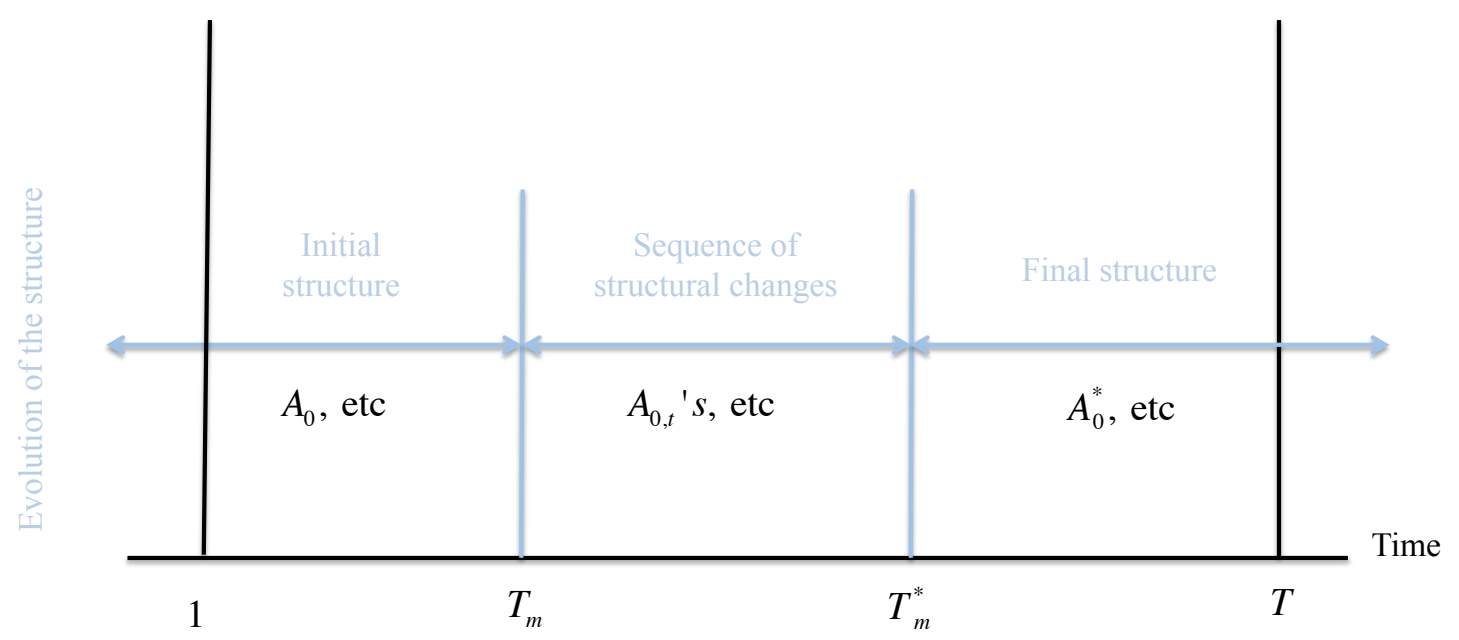


Figure 3: Timing of Structural Changes and Beliefs

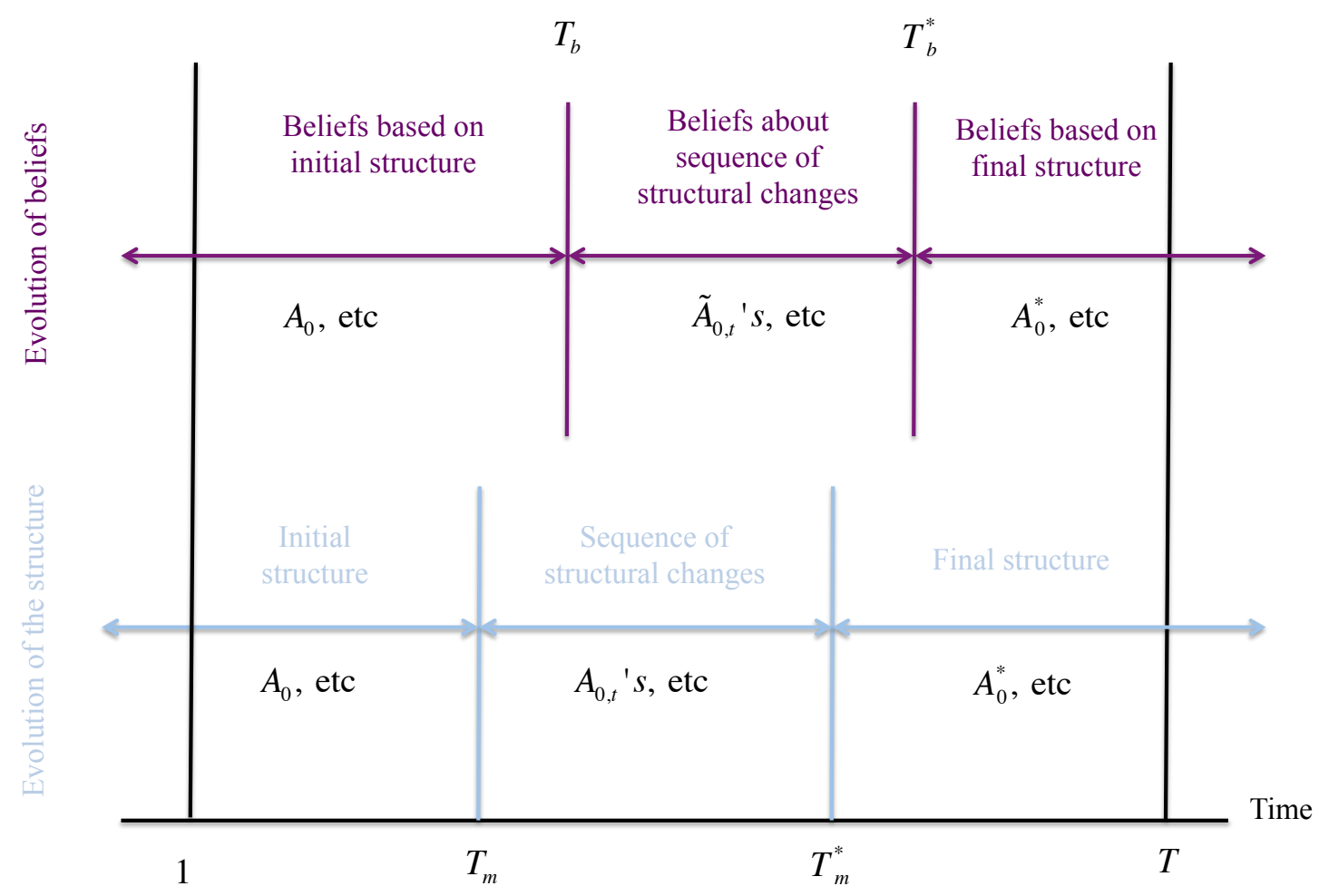


Figure 4: Impulse responses to a contractionary demand shock
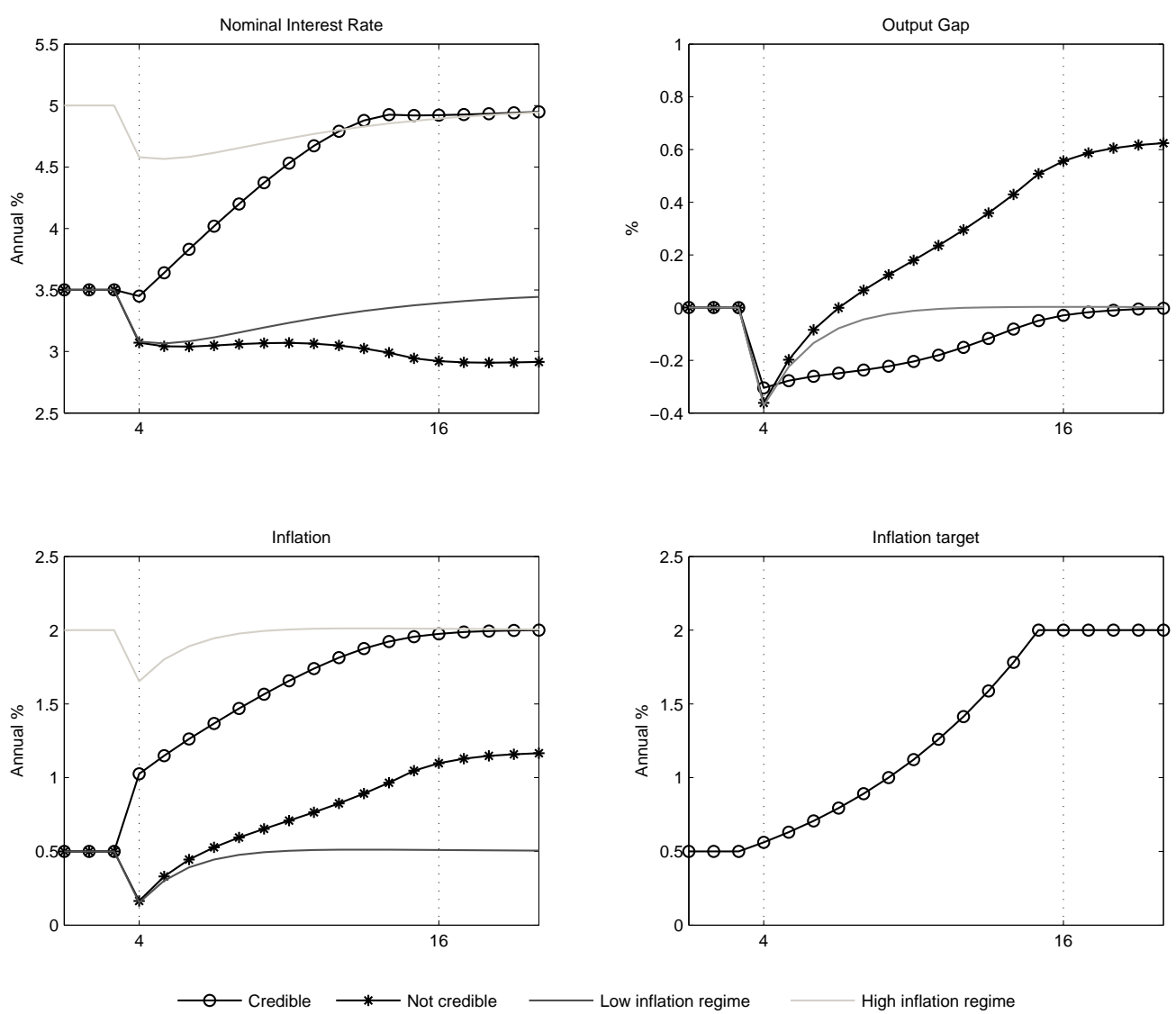


\section{List of Tables}

1 Parameter values for Experiment $1 \ldots \ldots \ldots 35$

2 Posterior Estimates . . . . . . . . . . . . . . . . . . . . 36 
Table 1: Parameter values for Experiment 1

\begin{tabular}{lll}
\hline Structural parameters & & Monetary policy rule parameter \\
$\rho_{r}$ & 0.9 & Monetary policy rule parameter \\
$\rho_{\pi}$ & 0.4 & Monetary policy rule parameter \\
$\rho_{g}$ & 0.1 & Monetary policy rule parameter \\
$\rho_{x}$ & 0.05 & Household's discount factor \\
$\beta$ & 0.9975 & Slope of the Phillips curve \\
$\psi$ & 0.1 & Frisch elasticity of labour supply \\
$\omega$ & 0.1 & Persistence of preference shock \\
$\rho_{a}$ & 0.85 & Persistence of supply shock \\
$\rho_{e}$ & 0.85 & Growth rate of labour augmenting TFP \\
$\bar{g}$ & 0.005 & Initial inflation target \\
$\pi^{(1)}$ & 0.00125 & Final inflation target \\
$\pi^{(2)}$ & 0.005 & Nominal interest rate \\
$\bar{r}^{(j)}$ & $\bar{\pi}^{(j)}+\bar{g}+\ln \beta$ & \\
\hline Timing of structural changes & 4 & Date new policy starts \\
$T_{m}$ & 16 & Date final structure is in place \\
$T_{m}^{*}$ &
\end{tabular}


Table 2: Posterior Estimates

\begin{tabular}{|c|c|c|c|c|c|c|}
\hline Parameter & $\begin{array}{l}\text { Posterior } \\
\text { Mode }\end{array}$ & $\begin{array}{l}\text { Posterior } \\
\text { Mean }\end{array}$ & \multicolumn{2}{|c|}{$\begin{array}{l}90 \text { per cent } \\
\text { C. I. }\end{array}$} & $\begin{array}{l}\text { Prior } \\
\text { Distribution } \dagger\end{array}$ & $\begin{array}{l}\text { Prior } \\
\text { Mean }\end{array}$ \\
\hline \multicolumn{7}{|c|}{ Standard Deviations } \\
\hline$\sigma_{r}$ & .0017 & .0017 & .0014 & .0019 & Inv. Gamma $(a, b)$ & .001 \\
\hline$\sigma_{a}$ & .0135 & .0142 & .0108 & .0186 & Inv. Gamma $(2.25,1.25)$ & .010 \\
\hline$\sigma_{e}$ & .0011 & .0011 & .0009 & .0012 & Inv. Gamma $(a, b)$ & .001 \\
\hline$\sigma_{z}$ & .0053 & .0053 & .0045 & .0060 & Inv. Gamma $(a, b)$ & .001 \\
\hline$\sigma_{\pi^{*}}$ & .0008 & .0009 & .0007 & .0010 & Inv. Gamma $(a, b)$ & .001 \\
\hline$\sigma_{\eta}$ & .0005 & .0005 & .0003 & .0007 & Inv. Gamma $(a, b)$ & .001 \\
\hline$\gamma$ & 2.250 & 2.283 & 2.070 & 2.511 & Normal $(1, .5)$ & 1.0 \\
\hline \multicolumn{7}{|c|}{ Structural Parameters } \\
\hline$\rho_{r}$ & .8366 & .8309 & .7792 & .8797 & $\operatorname{Beta}(c, c)$ & .5 \\
\hline$\rho_{\pi}$ & .8595 & .8785 & .6044 & 1.196 & Normal $(.5, .5)$ & .5 \\
\hline$\rho_{g}$ & .2849 & .2973 & .2125 & .3924 & Normal $(.2, .2)$ & .2 \\
\hline$\rho_{\pi}^{\prime}$ & .6538 & .6626 & .5471 & .8017 & Normal $(.5, .5)$ & .5 \\
\hline$\rho_{g}^{\prime}$ & .2639 & .2697 & .2120 & .3343 & Normal $(.2, .2)$ & .2 \\
\hline$\rho_{a}$ & .9075 & .9058 & .8735 & .9350 & $\operatorname{Beta}(c, c)$ & .5 \\
\hline$\rho_{e}$ & .2229 & .2308 & .1036 & .3821 & Beta $(c, c)$ & .5 \\
\hline$\rho_{\eta}$ & .8257 & .8099 & .7065 & .8914 & Beta $(c, c)$ & .5 \\
\hline$\rho^{*}$ & .9624 & .9594 & .9386 & .9745 & $\operatorname{Beta}(c, c)$ & .5 \\
\hline$g$ & .0042 & .0043 & .0034 & .0051 & Uniform $(0,0.02)$ & .01 \\
\hline$\pi$ & .0116 & .0113 & .0060 & .0161 & Uniform $(0,0.02)$ & .01 \\
\hline$\pi^{\prime}$ & .0066 & .0063 & .0045 & .0077 & Uniform $(0,0.02)$ & .01 \\
\hline
\end{tabular}

